\title{
Laparoscopic Indocyanine Green Sentinel Lymph Node Mapping in Endometrial Cancer
}

\author{
Andrea Papadia, MD, PhD ${ }^{1}$, Sara Imboden, $\mathrm{MD}^{1}$, Franziska Siegenthaler, $\mathrm{MD}^{1}$, Maria Luisa Gasparri, $\mathrm{MD}^{1,2}$, \\ Stefan Mohr, MD ${ }^{1}$, Susanne Lanz, $\mathrm{MD}^{1}$, and Michael D. Mueller, MD ${ }^{1}$ \\ ${ }^{1}$ Department of Obstetrics and Gynecology, University Hospital of Berne and University of Berne, Berne, Switzerland; \\ ${ }^{2}$ Department of Gynecology and Obstetrics, "Sapienza" University of Rome, Rome, Italy
}

\begin{abstract}
Background. In endometrial cancer (EMCA), indocyanine green (ICG) sentinel lymph node (SLN) mapping has been reported, mainly in conjunction with robotic surgery. Objective. We aimed to evaluate detection rates, sensitivity, and false negative (FN) rate of laparoscopic ICG SLN mapping in EMCA, and to evaluate differences in surgical outcomes between patients subjected to SLN biopsy only versus lymphadenectomy.

Methods. A retrospective analysis of EMCA patients undergoing ICG SLN mapping \pm pelvic (PLND) and/or para-aortic lymphadenectomy (PALND) was performed. Detection rates were calculated for the entire cohort. Sensitivity and FN rates were calculated for patients undergoing lymphadenectomy after SLN mapping, and surgical outcome was compared among patients undergoing SLN mapping only versus lymphadenectomy.

Results. Of 75 patients, 33 underwent SLN mapping and 42 underwent SLN mapping followed by PLND/PALND. Overall and bilateral detection rates were $96 \%$ (72/75) and $88 \%$ (66/75), respectively, and the median number of removed SLNs, pelvic non-SLNs (NSLN) and para-aortic NSLNs was 3, 27, and 19, respectively. With a FN rate of $8.3 \%$, only one patient had bilateral FN SLNs and a metastatic para-aortal NSLN. Estimated blood loss (EBL) and operative (OR) time were significantly lower in patients undergoing SLN mapping only. No differences in
\end{abstract}

Dr. Andrea Papadia and Dr. Sara Imboden have contributed equally to this work.

(C) The Author(s) 2016. This article is published with open access at Springerlink.com

First Received: 11 October 2015;

Published Online: 20 January 2016

A. Papadia, MD, PhD

e-mail: andrea.papadia@insel.ch complication rates between patients undergoing SLN mapping only and patients undergoing lymphadenectomy were recorded.

Conclusions. Laparoscopic ICG SLN mapping has excellent overall and bilateral detection rates and a low FN rate. Compared with lymphadenectomy, SLN biopsy is associated with significantly lower EBL and shorter OR time.

Since its definition in 1988, surgical staging in endometrial cancer (EMCA) has been controversial. ${ }^{1}$ Given the low risk of lymph node metastases in early-stage disease, it is believed that performing a pelvic (PLND) and para-aortic lymphadenectomy (PALND) in every patient is harmful rather than helpful. Various methods have been investigated to identify which patient benefit the most from surgical staging. The most widespread method to triage patients to surgical staging is via intraoperative identification of pathologic risk factors at frozen section ${ }^{2-6}$; however, frozen section is limited in that it provides only the risk of lymph node metastases. Thus, in patients intraoperatively identified as low risk, no information on lymph node status has been gathered.

Recently, interest has shifted from an intraoperative evaluation and an all-or-nothing approach to sentinel lymph node (SLN) mapping. ${ }^{7,8}$ Although SLN mapping is well-established in other tumors, in National Comprehensive Cancer Network (NCCN) guidelines its application in EMCA was only recognized to be appropriate in $2014 .^{9}$ In gynecological cancers, SLN mapping with ${ }^{99} \mathrm{Tc}$, blue dye, and indocyanine green (ICG), in conjunction with a nearinfrared (NIR) fluorescence imaging system, has been reported. ${ }^{10-12}$ ICG seems to stand out over the other tracers with regard to detection rates. ${ }^{13,14}$ In gynecologic oncology, ICG SLN mapping has been reported, mainly in 
conjunction with a robotic platform. ${ }^{12,13}$ In the laparoscopic setting, its use has been reported by only two other groups in patients with endometrial and cervical cancer, and by our group in cervical cancer. ${ }^{14-18}$

The aim of this study was to evaluate overall and bilateral detection rates, sensitivity, and false negative (FN) rates of the laparoscopic ICG SLN mapping in EMCA. Furthermore, we sought to evaluate differences in surgical outcome between patients subjected to SLN biopsy only versus full lymphadenectomy.

\section{MATERIALS AND METHODS}

A retrospective analysis of all patients with EMCA or complex atypical hyperplasia (CAH) undergoing ICG SLN mapping at our Institution between December 2012 and September 2015 was performed. Data on SLN mapping were collected prospectively. The study was approved by the Institutional Review Board, and all patients signed informed consent.

Patients underwent SLN mapping followed by laparoscopic lymph node biopsy, total hysterectomy (TLH) and bilateral salpingo-oophorectomy (BSO). Routine preoperative work-up included a transvaginal sonogram, as well as a chest $\mathrm{x}$-ray for low-risk tumors and a computed tomography (CT) scan for high-risk tumors. Focused imaging was performed based on clinical suspicion, and SLN mapping was performed with intraoperative cervical ICG injection. Following a diagnostic laparoscopy, the cervix was injected submucosally, $1 \mathrm{~cm}$ deep in the stroma at the four cardinal points, with a total of $8 \mathrm{ml}$ of ICG. One vial of $25 \mathrm{mg}$ ICG powder $\left(\right.$ Pulsion $^{\circledR}$ ) was previously suspended with $5 \mathrm{ml}$ of sterile water. The fluorescent signal was then identified under NIR mode, and the SLNs excised and sent to permanent pathology. Frozen section analysis on the SLN was performed only in cases of suspicion for metastasis. Suspicious non-SLNs (NSLNs) were removed and sent for frozen section. At final histopathological analysis, a complete ultrastaging was performed in all cases [three slides of hematoxylin and eosin (H\&E) $200 \mathrm{um}$, immunohistochemistry (IHC) if negative]. After having completed the TLH, the uterus was sent to frozen section and, based on identification of tumor risk factors and clinical judgment, a laparoscopic PLND and/or PALND was performed. In our institution, patients with type 2 EMCA, or with type 1 poorly differentiated and deeply invasive tumors, undergo a PPALND. Patients with type 1 well-differentiated lesions confined to the inner half of the myometrium undergo a SLN biopsy only. All other patients undergo a PLND.

Clinicopathologic characteristics were evaluated using the basic descriptive statistics. Overall and bilateral detection rates, sensitivity, and FN rate of the SLN mapping were calculated using Fisher's exact test. The false positive rate was defined as zero. The overall detection rate was calculated by dividing the number of procedures in which at least one SLN was identified by the total number of procedures performed, and the bilateral detection rate was calculated by dividing the number of procedures in which at least one SLN was identified on each side of the pelvis by the total number of procedures performed. A true positive SLN was defined as a positive SLN identified with histopathological techniques, independent of regional lymph node status, while an FN SLN mapping was defined as a bilateral negative SLN in combination with a metastatic NSLN. The FN rate of the SLN mapping was calculated for patients who underwent at least a PLND after the SLN biopsy. Surgical data, including estimated blood loss (EBL), operative (OR) time, and intra- and postoperative complication rates, were compared among the group of patients undergoing SLN mapping only and those undergoing a full lymphadenectomy after the SLN biopsy, using the unpaired $t$ test. To reduce the bias possibly linked to additional radical procedures, patients undergoing radical hysterectomy (RLH), radical colpectomy, or other non-EMCA concomitant-related surgeries were excluded from this analysis. Statistical analyses were performed using the $\mathrm{R}$ software (version 3.1.0). All $p$ values were two-sided, and $p$ values $<0.05$ were considered statistically significant.

\section{RESULTS}

Seventy-five patients were included in the study; their clinicopathologic characteristics are summarized in Table 1.

A TLH/BSO and SLN biopsy was performed on 33 patients. Of the remaining 42 patients, 13 underwent a PLND and 29 underwent a PPALND. Of the patients undergoing a full retroperitoneal staging, two also underwent an omentectomy. Three patients underwent an RLH due to suspicion of parametrial invasion on preoperative MRI in two cases, and vaginal involvement requiring a colpectomy in one case; one of these patients underwent a hemicolectomy for a concomitant colon cancer. All patients undergoing an RLH underwent a PPALND after the SLN biopsy. Conversion to laparotomy occurred in one case, secondary to macroscopic metastatic lymph node involvement. The median OR time was $220 \mathrm{~min}$, and the median EBL was $200 \mathrm{ml}$. Three intraoperative complications occurred, including one severe skin emphysema with hypercapnia requiring an intensive care unit (ICU) admission, one bladder lesion occurring in a stage IIIB patient undergoing a laparoscopic RLH and radical colpectomy, 
TABLE 1 Clinicopathologic characteristics of patients

\begin{tabular}{|c|c|}
\hline \multicolumn{2}{|l|}{ Patients $(N=75)$} \\
\hline Median age, years (range) & $65(38-89)$ \\
\hline Median BMI (range) & $27.2(17.4-47)$ \\
\hline \multicolumn{2}{|l|}{ FIGO stage } \\
\hline $\begin{array}{l}0 \text { (intraepithelial neoplasia, } \\
\text { complex hyperplasia) }\end{array}$ & $2(2.7)$ \\
\hline I & $53(70.7)$ \\
\hline II & $5(6.6)$ \\
\hline III & $14(18.7)$ \\
\hline IV & $1(1.3)$ \\
\hline \multicolumn{2}{|l|}{ Histology } \\
\hline $\begin{array}{l}\text { Complex atypical hyplerplasia/ } \\
\text { intraepithelial neoplasia }\end{array}$ & $2(2.7)$ \\
\hline Endometrioid & $66(88.0)$ \\
\hline Serous & $2(2.7)$ \\
\hline Clear cell & $1(1.3)$ \\
\hline Carcinosarcoma & $4(5.3)$ \\
\hline \multicolumn{2}{|l|}{ Grade } \\
\hline 1 & $24(32.9)$ \\
\hline 2 & $30(41.1)$ \\
\hline 3 & $19(26.0)$ \\
\hline \multicolumn{2}{|l|}{ Lymphovascular space invasion } \\
\hline Present & $17(22.7)$ \\
\hline Absent & $58(77.3)$ \\
\hline \multicolumn{2}{|l|}{ Myometrial invasion } \\
\hline Absent & $2(2.7)$ \\
\hline$<50 \%$ & $46(61.3)$ \\
\hline$>50 \%$ & $27(36.0)$ \\
\hline Patients with lymph node metastases & $11(14.7)$ \\
\hline \multicolumn{2}{|l|}{ Surgical lymph node assessment } \\
\hline SLN biopsy only & $33(44.0)$ \\
\hline SLN biopsy + PLND & $13(17.3)$ \\
\hline SLN biopsy + PLND + PALND & $29(38.7)$ \\
\hline Median number of PLNs (range) & $27(10-48)$ \\
\hline Median number of PALNDs (range) & $19(9-56)$ \\
\hline \multicolumn{2}{|l|}{ Operations performed } \\
\hline Hysterectomy + BSO & $33(44.0)$ \\
\hline Hysterectomy + BSO + PLND & $13(17.4)$ \\
\hline Hysterectomy + BSO + PLND + PALND & $26(34.7)$ \\
\hline $\begin{array}{l}\text { Radical hysterectomy + radical } \\
\text { colpectomy + BSO + PLND + PALND }\end{array}$ & $1(1.3)$ \\
\hline $\begin{array}{l}\text { Radical hysterectomy + hemicolectomy } \\
+ \text { BSO + PLND + PALND }\end{array}$ & $1(1.3)$ \\
\hline $\begin{array}{l}\text { Radical hysterectomy + BSO + PLND } \\
+ \text { PALND }\end{array}$ & $1(1.3)$ \\
\hline +Laparotomy & $1(1.3)$ \\
\hline +Omentectomy & $2(2.6)$ \\
\hline
\end{tabular}

Data are expressed as $n(\%)$ unless otherwise specified

$B M I$ body mass index, FIGO International Federation of Gynecology and Obstetrics, $S L N$ sentinel lymph node, $P L N D$ pelvic lymph node dissection, $P A L N D$ para-aortic lymph node dissection, $B S O$ bilateral salpingo-oophorectomy, PLNS pelvic lymph nodes
TABLE 2 Lymph node data

\begin{tabular}{ll}
\hline Overall SLN detection & $72 / 75(96.0)$ \\
Bilateral SLN detection & $66 / 75(88.0)$ \\
Unilateral SLN detection & $6 / 75(8.0)$ \\
No SLN detection & $3 / 75(4.0)$ \\
Median number of SLNs/patient (range) & $3(0-11)$ \\
Patients with lymph node metastasis & $11 / 75(14.7)$ \\
Macrometastasis & $7 / 11(63.6)$ \\
Micrometastasis & $4 / 11(36.4)$ \\
Isolated tumor cells & 0
\end{tabular}

Data in parentheses represent percentages unless otherwise stated $S L N$ sentinel lymph node

and one obturator nerve lesion. No ICG injection-related complications were registered. Two major postoperative complications occurred: one small bowel perforation requiring a relaparotomy in a patient undergoing a full laparoscopic surgical staging, and a large bowel anastomosis breakdown requiring a relaparotomy in the patient undergoing a hemicolectomy.

The median number of SLNs removed was three (range $0-11$ ), and overall and bilateral SLN detection rates were $96 \%$ (72/75) and $88 \%(66 / 75)$, respectively. Data on SLN mapping are summarized in Table 2. In two of the three patients in whom the SLN mapping failed, the pelvis had been previously irradiated, secondary to bladder cancer and pelvic lymphoma, respectively. By excluding these patients from the analysis, these rates rise to $98.6 \%(72 / 73)$ and $90.4 \%$ (66/73), respectively. Every patient in whom at least one SLN could be identified mapped in the pelvis. In $5 \%(4 / 75)$ of cases, additional SLNs were identified in the para-aortic region; no isolated para-aortic SLN was detected. The distribution of the SLNs is demonstrated in Fig. 1. Of the patients considered to be at low risk at frozen section, SLNs were metastatic in two cases.

Overall, $14.7 \%(11 / 75)$ of patients had lymph node metastases and, in all these cases, an SLN was detected bilaterally. Pathological evaluation of the SLNs showed micrometastases in four patients, all of which were diagnosed at H\&E. Of the 42 patients in whom a PLND/ PPALND was performed, one FN mapping occurred: both the bilateral SLNs in the pelvis and all the pelvic lymph nodes were negative, whereas two para-aortic NSLNs had metastatic disease. In another patient, four of five SLNs were true positive in the right hemipelvis, whereas one SLN in the left pelvis was FN as one NSLN had metastatic disease. The calculated sensitivity of the SLN mapping was $91.7 \%$; the FN rate was $8.3 \%$.

Thirty-three patients underwent a TLH/BSO and SLN biopsy, whereas 39 patients underwent the same surgical procedures followed by a PLND/PPALND. EBL and OR time were significantly lower among patients undergoing 


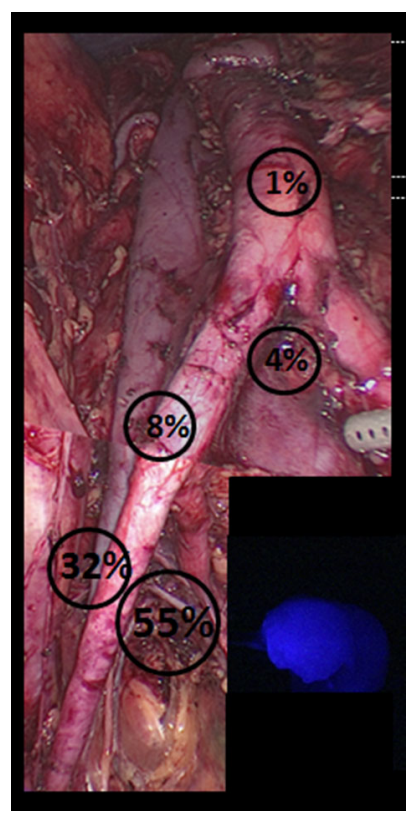

FIG. 1 Anatomic distribution of sentinel lymph node. Obturator fossa $55 \%$, external iliac artery $32 \%$, common iliac artery $8 \%$, aortic bifurcation $4 \%$, para-aortic area $1 \%$

an SLN mapping only compared with patients undergoing a full lymphadenectomy (200 $\mathrm{ml}$ vs. $300 \mathrm{ml}, p<0.0001$; $150 \mathrm{~min}$ vs. $300 \mathrm{~min}, p<0.0001$ ), but no differences were observed among intra- and postoperative complications in the two groups.

\section{DISCUSSION}

Although two randomized trials failed to show any benefit in EMCA patients who underwent a retroperitoneal staging, lymph node assessment still represents the cornerstone of EMCA staging. ${ }^{19,20}$ Lymph node status still represents one of the most important prognostic factors based on which adjuvant treatment is tailored. ${ }^{21-24}$

Our overall and bilateral detection rates of $96 \%(72 / 75)$ and $88 \%$ (66/75), respectively, are among the highest reported in the literature. Furthermore, by excluding two patients who had been previously subjected to pelvic radiation, these rates rise to $98.6 \%(72 / 73)$ and $90.4 \%$ (66/73), respectively. We speculate that the radiation-induced fibrosis alters the lymphatic flow, thus interfering with the SLN mapping; therefore, pelvic radiation should be a contraindication to SLN mapping. Similarly, Plante et al. reported overall and bilateral detection rates of 96 and $88 \%$, with a negative predictive value of $98.7 \%$ per side in 50 patients with uterine malignancies undergoing a laparoscopic SLN mapping. ${ }^{15}$ More recently, Buda et al. compared the outcome of two different SLN mapping tracers in 81 women with uterine malignancies undergoing laparotomy or laparoscopic surgery. ${ }^{17}$ They reported higher overall and bilateral detection rates in patients undergoing SLN mapping with ICG compared with methylene blue. Their overall and bilateral detection rates and negative predictive value were 100,88 and $100 \%$, respectively, when ICG was used. This is in accordance with our previously published data in cervical cancer. ${ }^{14}$

We believe that the good results recorded in our series are largely attributed to the tracer used. Desai et al. reported an overall and bilateral detection rate of 86 and $52 \%$, respectively, using blue dye, ${ }^{10}$ while in the SENTIENDO study, overall and bilateral detection rates were 89 and $69 \%$, respectively, using a combination of ${ }^{99} \mathrm{Tc}$ and blue dye. ${ }^{11}$ Solima et al. reported an overall detection rate of $95 \%$ after hysteroscopic injection of ${ }^{99} \mathrm{Tc}$; no data on their bilateral detection rate are reported. ${ }^{25}$ With the uterus being a midline structure, it is crucial to have a high bilateral detection rate to avoid unilateral lymph node dissections. Barlin et al. of the Memorial Sloan Kettering Cancer Center (MSKCC), proposed an SLN mapping algorithm for EMCA in which, in addition to the SLNs, every suspected lymph node is biopsied and a monolateral PLND is performed in those patients who map in only one hemipelvis. ${ }^{26}$ A high bilateral detection rate leads to a reduced number of bilateral and unilateral lymphadenectomies, possibly reducing surgical morbidity. By applying the MSKCC SLN mapping algorithm to our cohort of patients, one and six patients would have undergone a bilateral and monolateral PLND, respectively.

When SLN mapping is performed with ICG and the robotic platform, overall and bilateral detection rates vary between 87 and 82 and 97 and $57 \%$ in the different series, respectively. ${ }^{27-31}$ Our results correlate well with these data, suggesting that the dye, rather than the robot, is accountable for it.

In our study, one patient with a stage IIIB EMCA underwent SLN mapping followed by full surgical staging. Although SLN mapping also proved to be reliable in this case, with the bilateral identification of true negative SLNs we do not recommend relying on the SLN biopsy only in patients with stages other than stage I.

Compared to other mapping protocols, we injected a higher concentration of ICG. According to a recent metaanalysis, a lower concentration of ICG may provide better performance of the SLN mapping. ${ }^{32}$ In our hands, our protocol worked well and in no case was the operative field excessively stained.

In our study, every patient in whom at least one SLN could be identified mapped in the pelvis. In $5 \%(4 / 75)$ of cases, additional SLNs were identified in the para-aortic region. No isolated para-aortic SLNs were detected. Although the cervical injection of the tracer is the most common injection site in EMCA SLN mapping, there are 
concerns that tumors located in the fundus may spread through other lymphatic routes directly to the para-aortic region. For this reason, some centers propose a hysteroscopic tracer injection. ${ }^{31,33}$ In our series, only one case presented an isolated para-aortic lymph node metastasis, representing $2.4 \%$ of the staged patients. This percentage is in accordance with the risk of isolated para-aortic metastasis reported by Mariani et al. in fully staged, highrisk EMCA patients, providing further evidence that cervical injection of the tracer represents a safe way to perform SLN mapping in EMCA. ${ }^{34}$

Finally, we demonstrated that SLN biopsy is associated with a shorter OR time and a lower EBL compared with full surgical staging. Although these results may seem trivial, they have not yet been reported in the literature. However, our unique cohort of patients, including those who have undergone a SLN biopsy only and patients who have undergone SLN biopsy followed by a full lymphadenectomy, allowed for such an analysis. Intra- and postoperative complication rates are low and do not differ between the two groups. These results confirm data from the literature showing that, in experienced hands, a full laparoscopic staging is a feasible procedure, with low complication rates. ${ }^{35}$ The acquisition of the skills to perform a full pelvic and para-aortic laparoscopic lymphadenectomy is long and steep. ${ }^{36}$ Although SLN mapping seems to be more intuitive and require less surgical skill, it is crucial that it is performed accurately in order to avoid running the risk of sampling the wrong lymph node. Furthermore, although it is debated whether or not a full lymphadenectomy should be performed in patients with metastatic SLNs, considering that the MSKCC SLN mapping algorithm should be applied, we believe that every physician who performs a laparoscopic SLN mapping should have the skills to perform a full retroperitoneal staging.

Limitations of the study include its retrospective nature and the relatively small sample size. On the other hand, the prospective SLN mapping data collection, the relatively large percentage of patients undergoing PLND/PALND, and the thoroughness of the lymphadenectomy, as testified by the large number of lymph nodes removed, signify the strengths of the study.

\section{CONCLUSIONS}

This is the largest existing series on laparoscopic ICG SLN mapping in EMCA. Laparoscopic ICG SLN mapping in EMCA is characterized by high overall and bilateral detection rates and low FN rates that compare favorably with those reported when a robotic platform is used, suggesting that the tracer, rather than the surgical approach, is responsible for the SLN mapping outcome.
Compared with full surgical staging, SLN biopsy is associated with significantly lower EBL and shorter OR time, but no difference in intra- and postoperative complications. In a dualistic model in which a lymphadenectomy is fully performed or omitted based on intraoperatively evaluated pathologic tumor characteristics, ICG SLN mapping appears to represent a solomonic solution that gathers important pathologic information at low surgical expense.

ACKNOWLEDGMENT No sources of funding were used to support the present investigation.

CONFLICT OF INTEREST Andrea Papadia, Sara Imboden, Franziska Siegenthaler, Maria Luisa Gasparri, Stefan Mohr, Susanne Lanz, and Michael D. Mueller declare no conflicts of interest.

OPEN ACCESS This article is distributed under the terms of the Creative Commons Attribution 4.0 International License (http:// creativecommons.org/licenses/by/4.0/), which permits unrestricted use, distribution, and reproduction in any medium, provided you give appropriate credit to the original author(s) and the source, provide a link to the Creative Commons license, and indicate if changes were made.

\section{REFERENCES}

1. Creasman WT. FIGO stages: 1988 revisions. Gynecol Oncol. 1989;35(7):125-127.

2. Kumar S, Medeiros F, Dowdy SC, et al. A prospective assessment of the reliability of frozen section to direct intraoperative decision making in endometrial cancer. Gynecol Oncol. 2012;127:525-31.

3. Sala P, Morotti M, Menada MV, et al. Intraoperative frozen section risk assessment accurately tailors the surgical staging in patients affected by early-stage endometrial cancer: the application of 2 different risk algorithms. Int $J$ Gynecol Cancer. 2014;24:1021-6.

4. Papadia A, Azioni G, Brusacà B, et al. Frozen section underestimates the need for surgical staging in endometrial cancer patients. Int J Gynecol Cancer. 2009;19(9):1570-3.

5. Morotti M, Menada MV, Moioli $M$, et al. Frozen section pathology at time of hysterectomy accurately predicts endometrial cancer in patients with preoperative diagnosis of atypical endometrial hyperplasia. Gynecol Oncol. 2012;125(3):536-40.

6. Laufer J, Scasso S, Papadia A, et al. Association between tumor diameter and lymphovascular space invasion among women with early-stage endometrial cancer. Int $J$ Gynaecol Obstet. 2013;123:142-5.

7. Abu-Rustum NR. The increasing credibility of sentinel lymph node mapping in endometrial cancer. Ann Surg Oncol. 2013;20:353-4.

8. Kitchener HC. Sentinel-node biopsy in endometrial cancer: a win-win scenario? Lancet Oncol. 2011;12:413-4.

9. Version 2.2015: NCCN Clinical Practice Guidelines in Oncology

10. Desai PH, Hughes $\mathrm{P}$, Tobias DH, et al. Accuracy of robotic lymph node detection (RSLND) for patients with endometrial cancer (EC). Gynecol Oncol. 2014;135:196-200.

11. Ballester M, Dubernard G, Lécuru F, et al. Detection rate and accuracy of sentinel-node biopsy in early stage endometrial 
cancer: a prospective multicentre study. Lancet Oncol. 2011;12:469-76.

12. Rossi EC, Ivanova A, Boggess JF. Robotically assisted fluorescence-guided lymph node mapping with ICG for gynecologic malignancies: a feasibility study. Gynecol Oncol. 2012;124: 78-82.

13. Tanner EJ, Sinno AK, Stone RL, et al. Factors associated with successful bilateral sentinel lymph node mapping in endometrial cancer. Gynecol Oncol. 2015;138:542-7.

14. Imboden S, Papadia A, Nauwerk M, et al. A comparison of radiocolloid and indocyanine green fluorescence imaging, sentinel lymph node mapping in patients with cervical cancer undergoing laparoscopic surgery. Ann Surg Oncol. 2015;22:4198-203.

15. Plante M, Touhami O, Trinh XB, et al. Sentinel node mapping with indocyanine green and endoscopic near-infrared fluorescence imaging in endometrial cancer. A pilot study and review of the literature. Gynecol Oncol. 2015;137:443-7.

16. Touhami O, Trinh XB, Gregoire J, et al. Predictors of non-sentinel lymph node (non-SLN) metastasis in patients with sentinel lymph node (SLN) metastasis in endometrial cancer. Gynecol Oncol. 2015;138:41-5.

17. Buda A, Di Martino G, Vecchione F, et al. Optimizing strategies for sentinel lymph node mapping in early-stage cervical and endometrial cancer: comparison of real-time fluorescence with indocyanine green and methylene blue. Int $J$ Gynecol Cancer. 2015;25(8):1513-8.

18. Buda A, Bussi B, Di Martino G, et al. Sentinel lymph node mapping with near-infrared fluorescent imaging using Indocyanine green: a new tool for laparoscopic platform in patients with endometrial and cervical cancer. J Minim Invasive Gynecol. doi:10.1016/j.jmig.2015.09.022

19. Panici PB, Basile S, Maneschi $\mathrm{F}$ et al. Systematic pelvic lymphadenectomy vs no lymphadenectomy in early-stage endometrial carcinoma: randomized clinical trial. J Natl Cancer Inst. 2008;100(23): 1707-16.

20. ASTEC Study Group, Kitchener H, Swart AM, et al. Efficacy of systematic pelvic lymphadenectomy in endometrial cancer (MRC ASTEC trial): a randomised study. Lancet. 2009;373:125-136.

21. Simpkins F, Papadia A, Kunos C, et al. Patterns of recurrence in stage I endometrioid endometrial adenocarcinoma with lymphovascular space invasion. Int J Gynecol Cancer. 2013;23:98-104.

22. Landrum LM, Nugent EK, Zuna RE, et al. Phase II trial of vaginal cuff brachytherapy followed by chemotherapy in early stage endometrial cancer patients with high-intermediate risk factors. Gynecol Oncol. 2014;132:50-4.

23. Bogani G, Dowdy SC, Cliby WA, et al. Role of pelvic and paraaortic lymphadenectomy in endometrial cancer: current evidence. Obstet Gynaecol Res. 2014;40:301-11.
24. Sharma C, Deutsch I, Lewin SN, et al. Lymphadenectomy influences the utilization of adjuvant radiation treatment for endometrial cancer. Am J Obstet Gynecol. 2011;205:562.e1-9

25. Solima E, Martinelli F, Ditto A, et al.Diagnostic accuracy of sentinel node in endometrial cancer by using hysteroscopic injection of radiolabeled tracer. Gynecol Oncol. 2012;126:41923.

26. Barlin JN, Khoury-Collado F, Kim CH, et al. The importance of applying a sentinel lymph node mapping algorithm in endometrial cancer staging: beyond removal of blue nodes. Gynecol Oncol. 2012;125:531-5.

27. How J, Gotlieb WH, Press JZ, et al. Comparing indocyanine green, technetium, and blue dye for sentinel lymph node mapping in endometrial cancer. Gynecol Oncol. 2015;137:436-42.

28. Sinno AK, Fader AN, Roche KL, Giuntoli RL II, Tanner EJ. A comparison of colorimetric versus fluorometric sentinel lymph node mapping during robotic surgery for endometrial cancer. Gynecol Oncol. 2014;134:281-6.

29. Rossi EC, Jackson A, Ivanova A, Bogges JF. Detection of sentinel nodes for endometrial cancer with robotic assisted fluorescence imaging: cervical versus hysteroscopic injection Int J Gynecol Cancer. 2013;23:1704-11.

30. Holloway RW, Bravo RA, Rakowski JA, et al. Detection of sentinel lymph nodes in patients with endometrial cancer undergoing robotic-assisted staging: a comparison of colorimetric and fluorescence imaging. Gynecol Oncol. 2012;126:25-9.

31. Perrone AM, Casadio P, Formelli G, et al. Cervical and hysteroscopic injection for identification of sentinel lymph node in endometrial cancer. Gynecol Oncol. 2008;111:62-7.

32. Xiong L, Gazyakan E, Yang W, et al. Indocyanine green fluorescence-guided sentinel node biopsy: a meta-analysis on detection rate and diagnostic performance. Eur J Surg Oncol. 2014;40:843-9.

33. Ditto A, Martinelli F, Bogani G, et al. Sentinel node mapping using hysteroscopic injection of indocyanine green and laparoscopic near-infrared fluorescence imaging in endometrial cancer staging. J Minim Invasive Gynecol. 2015;22:132-3

34. Kumar S, Podratz KC, Bakkum-Gamez JN, et al. Prospective assessment of the prevalence of pelvic, paraaortic and high paraaortic lymph node metastasis in endometrial cancer. Gynecol Oncol. 2014;132:38-43.

35. Papadia A, Remorgida V, Salom EM, Ragni N. Laparoscopic lymphadenectomy in gynecologic oncology: a review. $J$ Am Assoc Gynecol Laparosc. 2004;11:297-306

36. Occelli B, Narducci F, Lanvin D, LeBlanc E, Querleu D. Learning curves for transperitoneal laparoscopic and extraperitoneal endoscopic paraaortic lymphadenectomy. J Am Assoc Gynecol Laparosc. 2000;7(1):51-3. 